\title{
First time expectant fathers' perspectives on breastfeeding
}

\begin{abstract}
Background: Mothers who have a supportive and encouraging partner are more likely to plan to breastfeed for a longer duration.

Objective: To explore the first time expectant fathers attitudes toward breast feeding practices.

Methods: A descriptive cross sectional study was carried out among $n=157$ purposively selected first time expectant fathers attending antenatal clinics along with low risk term primigravidae at a tertiary care corporate maternity hospital, Cosmopolitan Bangalore city, Karnataka, India from June 2015 to July 2016. In accordance with Declaration of Helsinki, data was collected by The Iowa Infant Feeding Attitudes Scale (IIFAS). SPSS version 20 was used for analysis.
\end{abstract}

Results: In general men had a favourable attitude towards breast feeding $(51.6 \pm 6.98)$ but they did not articulate specific benefits of it. Most expectant fathers reported that breastfeeding is the ideal way to feed a baby, contains the perfect proportion of nutrients and provides babies with a wide range of health benefits. Plus, breastfeeding is a great way for a mother to bond with her baby physically and mentally. However $(57.2 \%)$ of them preferred formula feeding for the baby while mother is away and felt it as healthy as breast milk. Although the men were generally supportive of breastfeeding, majority did not support breastfeeding $(58 \%)$ in the public places. A few knew about the long term benefits of breast feeding on mother and infants. Majority $(82.4 \%)$ expressed a need for support and information from health personnel on how they could practically support their partner in promoting and enabling breastfeeding. A significant association found between their attitude and level of education and occupation at 0.05 levels.

Conclusion: Health professionals must provide men centric prenatal education to promote breast feeding practices. They should involve, include and support fathers, recognizing their importance in the breastfeeding relationship.

Keywords: first time expectant fathers, breast feeding, the iowa infant feeding attitudes scale (IIFAS)
Volume 6 Issue 2 - 2017

\section{Samia Saud Al Furaikh, Thilagavathy Ganapathy \\ King Saud bin Abdul-Aziz University for Health Sciences, Saudi} Arabia

Correspondence: Samia Saud Al Furaikh, College of Nursing, Al Ahsa (CON-A), King Saud bin Abdul-Aziz University for Health Sciences (www.ksau-hs.edu.sa), King Abdul-Aziz Hospital, Ministry of National Guard Health Affairs, Saudi Arabia,Tel 966570870289,Email alfuraikhsa@ngha.med.sa

Received: October 25, 2016 | Published: November 06, 2017

\section{Introduction}

The decline in the breastfeeding practices in India, has led to serious implications for infant health. ${ }^{1,2}$ National Family Health Survey of India (NFHS 4) report that, only $52.1 \%$ of the infants under six months are exclusively breastfed and among them only $42.8 \%$ are breastfed on day one. ${ }^{3}$ Studies have shown that although moderately high initiation rates of breastfeeding exist in India, the prevalence and duration of exclusive breast feeding is very low, due to various factors that are difficult to address through breastfeeding promotion interventions. ${ }^{4-6}$ Several authors ${ }^{7-9}$ assert that a focus on modifiable factors such as maternal, paternal and significant others infant feeding attitude may help to increase breastfeeding duration. In cosmopolitan city where women are geographically isolated from their own mothers or other family members, the support of fathers as breastfeeding advocates may be more influential. ${ }^{7-11}$ Informal breast feeding support by male partners are repeatedly mentioned in the literature as an important source of support for breastfeeding mothers than formal source by health personnel. ${ }^{10-13}$ Women have more contact, access, and interaction with their personal social structures than with healthcare providers, and research indicates that the new mother's male partner and mother are the two most influential sources of informal social support, but male partners are often excluded from the mother and baby feeding relationship. ${ }^{7,11,13}$ Expanding the motherinfant breast feeding initiative to a mother-father-infant feeding initiative recognizes the importance of the male partner in supporting and strengthening breastfeeding efforts and the impact that the informal support structure can have in promoting breastfeeding. ${ }^{10,11,13}$ Kristen Mitchell-Box et al., ${ }^{13}$ reported that the most significant factor for mothers to initiate bottle-feeding was the mother's perception of the father's attitude.

Enhancing social support for breastfeeding is one of the three main strategies identified by UNICEF and WHO for increasing breastfeeding rates. ${ }^{14}$ However, educating those in the social support structures of new mothers to encourage initiation and duration of breastfeeding could be very helpful in increasing breastfeeding rates, especially in low-income populations countries. ${ }^{15,16}$ Research ${ }^{16,17}$ with mothers identifies fathers as a primary source of support for the continuance of breast feeding. Mothers who have a partner who is supportive and encouraging are more likely to plan to breastfeed, ${ }^{11,12}$ breastfeed on discharge from hospital ${ }^{12}$ and to breastfeed for a longer duration. ${ }^{17}$ Specifically, higher levels of paternal support and encouragement are associated with greater maternal confidence to breastfeed ${ }^{16,18}$ and 
mothers whose partner is supportive report feeling more capable and competent in breastfeeding decisions and challenges. ${ }^{19,20}$ The father is a primary source of support to the breastfeeding mother and can influence or contribute to decision making regarding initiation, continuance, maternal breastfeeding confidence and weaning. However, little is known about the nature of this support and their attitude with regards to supporting breastfeeding. ${ }^{20}$ It is important to understand more fully the role of a father's attitude in the breast feeding process as it is, unlikely that any intervention designed to increase rates of breastfeeding will be successful without taking this into account. ${ }^{10-13}$ Emphasis has been placed on educating and promoting the importance of breastfeeding to new mothers, potentially finding new and innovative ways to encourage greater initiation and duration. ${ }^{17,18}$ However, educating and supporting male partner who support the mother is also an important step. ${ }^{19,20}$ Studies $^{21,22}$ revealed that breastfeeding mothers were less likely to discontinue breastfeeding if it was the preferred feeding method of the father. However, the perception of first time expectant men with regards to infant feeding practices remains unclear and ambiguous and not understood fully by the health professionals. Therefore, a better understanding of first time expectant fathers' attitude, meaningful involvement, engagement and education of towards breastfeeding process is conceptually important to devise programs and strategies to improve breast feeding practices.

\section{Materials \& methods}

As breast feeding attitudes differ by socio-cultural factors to ensure recruitment of a heterogeneous group, a descriptive cross sectional approach was undertaken at a corporate private maternity hospital, Cosmopolitan Bangalore city, Karnataka, India. Based on the (NFHS 4) report that, only $52.1 \%$ of the infants under six months are exclusively breastfed, the sample size was calculated with $95 \%$ CI, with desired margin error at 0.09 , the calculated sample size were $\mathrm{N}=96$. To overcome missing data and nonresponsive rate, a total of one hundred fifty seven $(n=157)$ first-time expectant fathers attending prenatal clinic from June 2015 to July 2016 was targeted to explore their attitudes towards breast feeding practices. The eligible criteria were;

i. First time expectant men, irrespective of sociodemographic variables such as age, education, occupation, income and type of family,

ii. Whose partners were low risk primigravidae with no medical, surgical, obstetrical and gynecological risk factors complicating pregnancy, and

iii. Willing to participate and give informed consent.

Exclusion criteria were those first time expectant fathers who had close relatives such as cousins, brothers or sisters or parents or friends who worked in an infant formula feeding manufacturing company.

\section{Measurements}

First time expectant fathers infant feeding attitude was measured in the baseline questionnaire using the Iowa Infant Feeding Attitudes Scale (IIFAS) of De la Mora et al., ${ }^{23}$ a 17 item scale which measures attitudes towards both breast and formula feeding with regards to the health and nutritional benefits, and the cost and convenience of each method on a 5-point Likert Scale ranging from (strongly agree, agree, neutral, disagree, and strongly disagree). It has eight statements favourable toward breastfeeding and the remaining nine (i.e. 1,
$2,4,6,8,10,11,14$, and 17) favourable toward formula feeding. Items that favoured formula feeding were reverse scored and a total attitude score was computed by means of an equally weighted sum of responses to the individual items. Total attitude scores could range from 17 (reflecting positive formula feeding attitudes) to a high of 85 (indicating attitudes that favoured breastfeeding). Content validity of the tool had been established by the experts and the content validity index was -CVI0.89. The IIFAS was pilot tested among 15 first time expectant men for usability and translated from English into Kannada using the forward backward method by professional native translators specialized in medicine and the reliability of this questionnaire yielded 0.85 with Cronbach's alpha.

\section{Ethical approval}

This study follows the ethical principles of The Declaration of Helsinki. Ethical permission was obtained from the Maternal Child health and family welfare department and clinical permission to conduct the study from the medical and nursing superintendent of the hospital. Informed consent was obtained from the participants and the confidentiality of the information were assured. Participants were not considered to be at risk of harm. The first time expectant fathers were informed that the duration of the interview would be approximately 30-40minutes. The interview was taken in a flexible manner, as one to one interview. They were informed that the written results of the study might be published, and that their identities would be kept confidential.

\section{Statistical analysis}

Statistical analysis was conducted using SPSS 20 (Chicago, III USA). Descriptive statistics -frequencies, mean scores and mean percentage were performed on all items and non-parametric Chi-square/ Fishers exact probability tests were used to find the association of fathers' attitudes towards infant feeding practices with sociodemographic variables. An alpha of less than 0.05 was considered significant in all tests.

\section{Results}

\section{Description of the study participants}

Of the 157 first time expectant fathers eligible to participate in the present study $\mathrm{N}=143$ consented to participate, seven withdrew after answering three to five questions and were excluded without replacement and the final sample constituted of $\mathrm{N}=136$. The mean age of first time expectant fathers was 26.4years (SD 3.3, range 24.0 32.0). With regard to educational level $(64.6 \%)$ of men had postgraduate education. The rest $(45.4 \%)$ had technical education. The majority of them $(84.8 \%)$ were private sectors employees, $(3.9 \%)$ in Govt sector and $(11.3 \%)$ were self-employed. Majority of them belonged to the upper middle socio economic strata of society. There was no significant association between the sociodemographic variables and the perspectives of fathers on breast feeding at 0.05 level of significance $\left(\chi^{2}=2.401 ; p>0.05\right)$.

\section{Expectant fathers perception on benefits of breast feeding}

About (55.4\%) expressed that breast milk is the ideal food for babies $(49.4 \%)$ agreed that breastfeeding is more convenient than formula feeding and $(48.4 \%)$ reported that breast milk is less expensive than formula. Nearly $(45.2 \%)$ agreed that breast milk is more easily 
digested than formula and half of them (56.2\%) agreed that babies who are breastfed would be healthier than formula-fed babies. Nearly one thirds $(47.8 \%)$ felt that breastfeeding increased mother-infant bonding and majority of fathers (56.8\%) agreed that formula feeding meant missing one of the great joys of motherhood (Table 1).

\section{Expectant fathers perception regarding formula feeding}

Majority (56.2\%) of first time expectant fathers felt that formula feeding is more convenient than breastfeeding. Nearly $57.2 \%$ preferred formula feeding as a better choice if a mother plans to work outside the home. Majority $52.8 \%$ disagreed that formula fed babies are more likely to be overfed than breast-fed babies and $52 \%$ felt that breastfed babies are more likely to be overfed than formula fed babies. Most of the first time expectant men $(49.8 \%)$ perceived that the nutritional benefits of breast milk last only until the baby is weaned from breast milk and $48.4 \%$ were ambiguous about its iron content (Table 1).

\section{Expectant fathers perception towards breast feeding in public places}

Nearly two thirds (58\%) of them were against breast feeding in public places and preferred formula feeding over breast feeding in public. Most of them (45\%) agreed that mother should not consume alcohol while breast feeding (Table 1).

Table I Socio demographic characteristics of the participants

\begin{tabular}{lll}
\hline Demographic Variables & Frequency & Percentage \\
\hline 1. Age in Years & & \\
A) Less than 28 & 49 & 65 \\
B) 28 and above & 48 & 35 \\
2. Education & & 45.4 \\
A) Technical & 88 & 64.6 \\
B) Graduate/Postgraduate & & \\
3. Occupation & 115 & 84.8 \\
A) Private sectors & 16 & 11.3 \\
B) Self- employed & 5 & 3.9 \\
C) Govt Sector & & \\
4. Income/ Month & 102 & 75 \\
A) Below 40.000 & 34 & 25 \\
B) Above 40,000 &
\end{tabular}

$\mathrm{N}=136$

Table 2 The lowa infant feeding attitude scale (IIFAS) questions

\begin{tabular}{lll}
\hline & Median & Percentage \\
\hline *1. The nutritional benefits of breast milk last only until the baby is weaned from breast milk. & 2.49 & 49.8 \\
*2. Formula feeding is more convenient than breastfeeding. & 2.81 & 56.2 \\
3. Breastfeeding increases mother-infant bonding. & 2.39 & 47.8 \\
*4. Breast milk is lacking in iron. & 2.42 & 48.4 \\
5. Formula fed babies are more likely to be overfed than are breast-fed babies. & 2.36 & 47.2 \\
*6. Formula feeding is the better choice if a mother plans to work outside the home. & 2.86 & 57.2 \\
7. Mothers who formula feed miss one of the great joys of motherhood. & 2.84 & 56.8 \\
*8. Women should not breastfeed in public places such as restaurants. & 2.9 & 58 \\
9. Babies fed breast milk are healthier than babies who are fed formula. & 2.81 & 56.2 \\
*10. Breastfed babies are more likely to be overfed than formula fed babies. & 2.6 & 52 \\
*11. Fathers feel left out if a mother breast- feeds. & 2.52 & 50.4 \\
12. Breast milk is the ideal food for babies. & 2.77 & 55.4 \\
13. Breast milk is more easily digested than formula. & 2.26 & 45.2 \\
*14. Formula is as healthy for an infant as breast milk. & 2.69 & 53.8 \\
15. Breastfeeding is more convenient than formula feeding. & 2.47 & 49.4 \\
16. Breast milk is less expensive than formula. & 2.42 & 48.4 \\
*17. A mother who occasionally drinks alcohol should not breastfeed her baby. & 2.25 & 45 \\
Overall mean & 2.578 & $51.6 \pm 6.98$ \\
\hline
\end{tabular}

*The items I, 2, 4, 6, 8, 10, II, 14, and 17 were reversed when calculating the score. 


\section{Discussion}

Unfortunately the concept of breast feeding is usually relegated to women and men are excluded from the intimacy of mother and infant feeding relationship. But fathers actually have tremendous potential to either facilitate or undermine the success of breastfeeding. ${ }^{20-22,24}$ If father perceives that breastfeeding is having a positive effects in the health and well-being of his child and as a high priority for his partner and child, this attitude will set the desired tone for achieving success. ${ }^{22,24}$ In this study $51.6 \%$ of the first time expectant men felt positive towards breast feeding practices, with most of them reflecting at least one benefit of breastfeeding. Fathers strongly believed that their infants will definitely be benefitted from being breastfed and that they would be healthy and free from illness as breast milk is ideal and natural food for them. Cost also featured very strongly in fathers positive reflections on breastfeeding. A number of fathers felt that, breastfeeding is an easy and convenient option and mothers need not have to worry about preparing bottles or running out of milk. These findings are in line with studies by Sherriff et al., ${ }^{22}$ \& Tohotoa et al., ${ }^{24}$ that fathers were interested in breastfeeding and wanted their infants to be breast fed for better health benefits and also to be involved in the breast feeding relationship.

The first hours and weeks with her new-born after childbirth is an overwhelming time for a new mother. ${ }^{16,25}$ An initial experience in the early postpartum period of breastfeeding is influential in a woman's determination to continue to breastfeed. Many women are unprepared for the learning process involved in establishing breastfeeding successfully, and without the right encouragement and support, ${ }^{16}$ many women fall short of the recommended six months of exclusive breastfeeding. ${ }^{25,26}$ Although many women intend to breastfeed before the birth of a child, sleep deprivation, the responsibilities of taking care of a new born, return to work, other children, and many other factors can affect breastfeeding initiation, exclusivity, and duration. . $^{615-17,25,26}$ Mothers who have a partner who is supportive and encouraging are more likely to plan to breastfeed for a longer duration. ${ }^{21,22,24}$ Rempel et al., ${ }^{27}$ in their study reported that fathers wanted to be a part of the breast feeding team to support their wives physically by sharing the household chores and emotionally boosting their confidence, to initiate and sustain breast feeding for a longer duration. Scott et al., ${ }^{21} \&$ Rempel et al., ${ }^{27}$ also reported that those mothers who receive support from their spouses usually opt for exclusive and optimum breastfeeding for their infants, and the chances of continuing this practice are higher. Parental attitudes are strong predictors of initiation, continuation and the duration of breastfeeding. ${ }^{20-22,27}$

Health professionals should acknowledge the role and contribution of the father in supporting breastfeeding and assist them to understand their role in promotion of breast feeding practices. ${ }^{28,29}$ Although the first time expectant fathers were positive towards breast feeding, nearly two thirds of them (57.2\%) perceived that formula feeding is more convenient than breast milk, and a better choice for mother when she goes out and it is as healthier $(53.8 \%)$ as breast feeding. Proliferation of advertisement on artificial infant feeding and posters displayed in the clinics, hospitals, portraying healthy, cuddly babies have provided a level of reassurance for expectant parents regarding their choice of infant feeding method. The rise of social media and networking sites present new challenges in monitoring the pervasiveness of messages promoting infant formula at the expense of the benefits of breastfeeding. Berry et al., ${ }^{30}$ who examined the influence of advertising of toddler milk products found that first time mothers perceived these advertisements to be for infant formula and believed the manufacturers' claims that formula could provide benefits similar to those normally attributed to breastfeeding based on this information.

Wider community promotion of breastfeeding guidelines, population health benefits and support of breastfeeding goals may help to establish breastfeeding as the normal choice of infant feeding and counter these messages to influence the attitudes of women of childbearing age, as well as their partners and other social supports. ${ }^{27-29}$ Without this general support, breastfeeding messages may be less effective, contributing to the difficulty mothers face in reaching the recommendations for exclusive breastfeeding. ${ }^{28,29}$ In the present study, although most men wanted their babies to be breast fed, most of them expressed that, the choice of infant feeding is completely mothers decision and they would agree and support in whatever they wanted to do. In Rempel et al., ${ }^{27}$ study whilst all fathers felt the mother had the final say regarding the decision to, and stop, breast feeding a number of fathers were still involved in decisions about whether and how long to breast feed. In Abu-Abbas et al., ${ }^{31}$ study in Jordan all fathers considered breastfeeding as the mothers responsibility, the mother had the final say regarding the decision to breastfeed and they have no role to play in it. Whilst fathers often report that they do not see themselves as being influential in their partner's feeding decisions, it is clear that mothers feel partner support is crucial, ${ }^{28,29,32}$ particularly in the form of validation both in the initial decision and in the on-going decision regarding mode of infant feeding. Although the men were generally supportive of breastfeeding, majority (58\%) of did not support public breastfeeding and reported that breastfeeding in public is them embarrassing or unacceptable. They felt that public breastfeeding was wrong in the context of witnessing it themselves and of being with their partners in a public breastfeeding situation. Several authors ${ }^{31-33}$ have found that whilst most fathers generally have positive attitudes towards breastfeeding some groups of parents have less positive attitudes. Avery et al., ${ }^{32}$ found that whilst both mothers and fathers attitudes to breast feeding were broadly favourable, attitudes to breast feeding in public was unfavourable. Mohamad et al. ${ }^{34}$ reported that fathers believed that breast feeding was unacceptable in public and majority of fathers felt that breast feeding even in in front of family members and friends were embarrassing and totally unacceptable practice. Similar findings was reported by Abu-Abbas et al., ${ }^{31}$ that fathers of both artificial milk and breast-feeding infants demonstrated highly negative attitude towards breast feeding in public and in front of family members. Consistent findings were reported by Taspinar et al., ${ }^{33}$ that half of the fathers felt uncomfortable about their wives breast feeding in public places such as busses, parks and taxis.

Research, ${ }^{33,34}$ shows that there are social stigmas surrounding breastfeeding in public. Many cultures consider the female breast primarily as a sexual organ, and therefore a private part of the body, which needs to be invisible in the public arena. ${ }^{31-35}$ Groleau et al., ${ }^{35}$ point to the urgent need for reintroducing the nutritional role of the breast into various social and public spaces including the medias. Reintroducing the normality of breastfeeding in visible public places through images and pictures of women of all ages, body types and styles would be a positive step toward making breastfeeding an infantfeeding type. In this study majority $(82.4 \%)$ expressed a need for support and specific and detailed information from health personnel on how they could practically support their partner in promoting and enabling breastfeeding. Fathers also reported wanting more specific and accessible information about the benefits of breastfeeding. 
Sheriff, ${ }^{19,22}$ asserts that fathers need to be involved in breast feeding education to support mothers in infant feeding practices. Tohotoa et al., ${ }^{24}$ suggested that breastfeeding education should be expanded to include fathers, particularly equipping them with the skills they need to support their partner through difficulties.

The study findings suggests that fathers are interested in breastfeeding and want to be involved with and support the mother through the breast feeding time. ${ }^{24,27,29}$ However, fathers often feel unprepared for the challenges breastfeeding can bring, ${ }^{27}$ unsure how to help ${ }^{29}$ or feel left out, helpless and excluded from the experience. ${ }^{13}$ Whilst both Tohotoa et al., ${ }^{24}$ and Sherriff et al., ${ }^{22}$ report that fathers want greater relevant information and practical advice about breast feeding that is specifically targeted for them, in order to know the best ways to help their breast feeding partner. Health professionals must involve, include them in education and support fathers, recognising their importance in the breastfeeding relationship. ${ }^{21,32}$ Breastfeeding education for fathers should not end with the prenatal education. Partners should have the self-efficacy to make breastfeeding work for as long as possible. Follow-up with the breastfeeding mother should include a follow-up with the father to monitor how he is handling the situation. . $^{28,36,37}$

\section{Implications for practice}

Health professionals must recognise the importance of father in breast feeding relationship and provide father focused flexible breastfeeding educational sessions during prenatal check up, to enhance their knowledge and self-efficacy to support breast feeding practices.

\section{Limitation}

As the fathers in the sample were from culturally diverse backgrounds of cosmopolitan Bangalore city, generalization to other areas is limited.

\section{Conclusion}

The findings show that expectant fathers had favourable attitude toward breast feeding, willing and enthusiastic to support their partners to initiate and continue breastfeeding. However, men wanted consistent support, encouragement and men friendly evidence-based breastfeeding information from the health personnel and wished to be recognised as important advocates to support their partners to begin and continue to breastfeed to meet the current WHO recommendations.

\section{Acknowledgements}

We are grateful to all the participants, their partners, midwives, obstetricians and medical and nursing superintendent of the study setting for their support and cooperation.

\section{Conflict of interest}

The author declares no conflict of interest.

\section{References}

1. Chandhiok N, Singh KhJ, Sahu D, et al. Changes in exclusive breastfeeding practices and its determinants in India, 1992-2006: analysis of national survey data. Int Breastfeed J. 2015;10:34.
2. Chinnasam B, Sundaramoorthy S, Sadasivam K, et al. Knowledge, attitude and practices of mothers regarding breastfeeding in a south Indian hospital. Biomed Pharmacol J. 2016;9(1):195-199.

3. National Family Health Survey (NFHS 4). India: International institute for population sciences; 2016.

4. Pandey D, Sardana P, Saxena A, et al. Awareness and attitude towards breastfeeding among two generations of Indian women: a comparative study. PLoS ONE. 2015;10(5); 0126575.

5. Maheswari E, Vishnu Bhat B, et al. Knowledge, attitude and practice of breastfeeding among postnatal mothers. Curr Pediatr Res. 2010;14(2):119 124.

6. Choudhary AM, Bankwar V, Choudhary A. Knowledge regarding breastfeeding and factors associated with its practice among postnatal mothers in central India. Int J Med Sci Public Health. 2015;4(7):973-976.

7. Meedya S, Fahy K, Kable A. Factors that positively influence breastfeeding duration to 6months: a literature review. Women Birth. 2010;23(4):135145 .

8. Karande S, Perkar S. Do fathers' attitudes support breastfeeding? a crosssectional questionnaire based study in Mumbai, India. Indian J Med Sci. 2012;66(1-2):30-39.

9. Tan KL. Factors associated with exclusive breastfeeding among infants under six months of age in peninsular Malaysia. Int Breastfeed $J$. $2011 ; 6(1): 2$.

10. Mithani Y, Premani ZS, Kurji Z, Exploring fathers' role in breastfeeding practices in the urban and semi urban settings of Karachi, Pakistan. $J$ Perinat Educ. 2015;24(4):249-260.

11. Wolfberg A, Michels K, Shields W, et al. Dads as breastfeeding advocates: results from a randomised controlled trial of an educational intervention. Am J Obstet and Gynecol. 2004;191(3):708-712.

12. Maycock BR, Scott JA, Hauck YL, et al. A study to prolong breastfeeding duration: design and rationale of the parent infant feeding initiative (PIFI) randomised controlled trial. BMC Pregnancy Childbirth. 2015;15:159.

13. Mitchell-Box K, Braun KL. Fathers' thoughts on breastfeeding and implications for a theory $\square$ based intervention. J Obstet Gynecol Neonatal Nurs. 2012;41(6):E41-50.

14. http://www.unicef.org/nutrition/index_breastfeeding.html.

15. Persad MD, Mensinger JL. Maternal breastfeeding attitudes: association with breastfeeding intent and socio-demographics among urban primiparas. J Community Health. 2008;33(2):53-60.

16. Scott JA, Landers MC, Hughes RM, et al. Factors associated with breastfeeding at discharge and duration of breastfeeding. J Paediatr Child Health. 2001;37(3):254-261.

17. Brown A, Lee M. Breastfeeding during the first year promotes satiety responsiveness in children aged $18-24$ months. Pediatr Obes. 2012;7(5):382-390.

18. Mannion CA, Hobbs AJ, McDonald SW, et al. Maternal perceptions of partner support during breastfeeding. Int Breastfeed J. 2013;8(1):4.

19. Sherriff N, Panton C, Hall V. A new model of father support to promote breastfeeding. Community Pract. 2014;87(5):20-24.

20. Powell K, Baic S. Partner support for breastfeeding: the need for health promotion. Journal of Human Nutrition and Dietetics. 2011;24(4):402402 . 
21. Scott JA, Binns CW, Oddy WH, et al. Predictors of breastfeeding duration: evidence from a cohort study. Pediatrics. 2006;117(4):e646-e655.

22. Sherriff N, Hall V, Panton C. Engaging and supporting fathers to promote breast feeding: a concept analysis. Midwifery. 2014;30(6):667-677.

23. De la Mora A, Russell DW, Dungy CI, et al. The Iowa Infant Feeding Attitude Scale: Analysis of reliability and validity. Journal of Applied Social Psychology. 1999;29(11):2362-2380.

24. Tohotoa J, Maycock B, Hauck Y, et al. Dads make a difference: an exploratory study of paternal support for breastfeeding in Perth, Western Australia. Int Breastfeed J. 2009;29:15.

25. Li R, Fein SB, Chen J, et al. Why mothers stop breastfeeding: mothers' self-reported reasons for stopping during the first year. Pediatrics. 2008;122(Suppl 2):S69-76.

26. Zhang K, Tang L, Wang H, et al. Why do mothers of young infants choose to formula feed in china? Perceptions of mothers and hospital staff. Int $J$ Environ Res Public Health. 2015;12(5):4520-4532.

27. Rempel L, Rempel J. The breastfeeding team: the role of involved fathers in the breastfeeding family. J Hum Lact. 2011;27(2):115-121.

28. Kylee NC, Roslyn CG, Colin WB. The influence of infant feeding attitudes on breastfeeding duration: evidence from a cohort study in rural Western Australia. International Breastfeeding Journal. 2015;10:25.

29. Brown A, Davies R. Fathers' experiences of supporting breastfeeding: challenges for breastfeeding promotion and education. Matern Child Nutr. 2014;10(4):510-526.

30. Berry N, Jones S, Iverson D. Toddler milk advertising in Australia: the infant formula ads we have when we don't have infant formula ads. In Ballantine P \& Finsterwalder J (Eds.), ANZMAC Annual Conference 2010: Australian and New Zealand Marketing Academy Conference 2010, Doing More with Less, Australia; 2010. p. 1-8.
31. Abu-Abbas MW, Kassab IM, Shelash IK. Fathers and breastfeeding process: determining their role and attitudes. European Scientific Journal. 2016;12(18):327-336.

32. Avery AB, Magnus JH. Expectant fathers' and mothers' perceptions of breastfeeding and formula feeding: a focus group study in three US cities. J Hum Lact. 2011;27(2):147-154.

33. Taşpinar A, Çoban A, Küçük M, et al. Fathers' knowledge about and attitudes towards breast feeding in Manisa, Turkey. Midwifery. 2013;29(6):653-660.

34. Mohamad N, Draman N, Muhamad R, et al. Knowledge and attitude towards exclusive breastfeeding practices among fathers who attend primary health care facilities in suburban, Malaysia. International Journal of Collaborative Research on Internal Medicine \& Public Health. 2015;7(7):154-163.

35. Groleau D, Sigouin C, D'Souza NA. Power to negotiate spatial barriers to breastfeeding in a western context: When motherhood meets poverty. Health Place. 2013;24:250-259.

36. Maycock B, Binns C, Dhaliwal S, et al. Education and support for fathers improves breastfeeding rates: a randomized controlled trial. Journal of Human Lactation. J Hum Lact. 2013;29(4):484-490.

37. Susin LR, Giugliani ER. Inclusion of fathers in an intervention to promote breastfeeding: impact on breastfeeding rates. J Hum Lact. 2008;24(4):386392. 